\title{
Chemotherapy and role of the proliferation marker Ki-67 in digestive neuroendocrine tumors
}

\author{
Eduardo Vilar, Ramón Salazar ${ }^{1}$, Jose Pérez-García, Javier Cortes, Kjell Öberg ${ }^{2}$ \\ and Josep Tabernero
}

\author{
Department of Medical Oncology, Vall d'Hebron University Hospital, Ps. Vall d'Hebron, 119-129, Barcelona 08035, Spain \\ ${ }^{1}$ Department of Medical Oncology, Institut Català d'Oncologia, Barcelona, Spain \\ ${ }^{2}$ Department of Endocrine Oncology, University Hospital, 75185 Uppsala, Sweden \\ (Requests for offprints should be addressed to E Vilar; Email: evilar@vhebron.net)
}

\begin{abstract}
Neuroendocrine tumors (NETs) of the digestive tract are a heterogeneous group of rare malignancies. Three major subgroups can be defined: pancreatic endocrine tumors, carcinoid tumors, and poorly differentiated gastroenteropancreatic NETs. Classically, digestive NETS have been considered to have an indolent course characterized for prolonged stabilizations or slow progressions, but there are clear differences in terms of aggressiveness, clinical course, and response to treatment among them. Retrospective studies have identified several clinicopathological and immunohistochemical factors as angioinvasion and proliferative index assessed by Ki-67 expression, which predict biological behavior and correlate with survival. Chemotherapy regimens based on the combination of several active drugs such as streptozocin, doxorubicin, 5-fluorouracil, dacarbazine, and temozolomide show low response rates, which sets the need to improve the results of the medical treatment of these malignancies. This review will analyze the role of Ki-67 in digestive NETs under a clinical perspective and will suggest future fields for development of this approach that enable a better patient selection for chemotherapy. Also a comprehensive review of the literature about chemotherapy in NETs is presented.
\end{abstract}

Endocrine-Related Cancer (2007) 14 221-232

\section{Introduction}

Neuroendocrine tumors (NETs) of the digestive tract include different subsets of malignancies, such as pancreatic endocrine tumors (PETs), carcinoid tumors (CTs), and other poorly differentiated gastroenteropancreatic NETs. They can also present themselves as liver metastasis with markers and histological features of neuroendocrine origin without a clear primary tumor with a conventional radiological work-up.

The WHO classification of 2000 divided neuroendocrine malignancies into three groups: well-differentiated NET, well-differentiated neuroendocrine carcinoma, and poorly differentiated neuroendocrine carcinoma. Distinction between groups relies on several pathological and clinical data such as invasion of muscularis propria, histological differentiation (well-differentiated versus poorly differentiated), tumor size $(<2$ vs $\geq 2 \mathrm{~cm})$, angioinvasion, Ki-67 index $(<2,>2$, and $>30 \%)$, biological behavior (benign, low-grade and high-grade malignant), presence of metastases, and presence of hormonal symptoms. However, a sub-classification according to tumor localization distinguished several origins: gastric, duodenum, ileum, appendix, colonrectum, and pancreas. In this review, we have included both well-differentiated pancreatic NETs and carcinomas under the term of PETs, and both well-differentiated NETs and carcinomas from any other localization of the gastrointestinal tract (including gastric, duodenum, upper jejunum, ileum, cecum, colon-rectum, and appendix tumors) under the term of CT. Poorly differentiated tumors are characterized by extensive angioinvasion, increased necrosis, nuclear atypia, and high mitotic activity measured by mitotic count or by $\mathrm{Ki}-67$. Although a clear cut-off value for Ki-67 has not been established, several authors placed it between 20 and 30\% (Kloppel et al. 2004, Rindi et al. 2006).

NETs are rare as they constitute $<2 \%$ of all gastrointestinal malignancies (Oberg et al. 2004b); 
CTs are the most frequently occurring NETs with an adjusted incidence rate of approximately four cases per 100000 population per year, which appears to have increased over the past 30 years (Modlin et al. 2003). Incidence of PETs is estimated from indirect data based on the largest series reported (Jensen \& Norton 1995).

Most of the cases have low growth rates with survival times longer than other gastrointestinal tumors, site by site. Thus, use of chemotherapy in cases of unresectable locoregional or advanced disease is usually preferred only after treatment with locoregional techniques and biological therapies have failed.

Several studies have tried to better define the prognosis of patients using several immunohistochemistry markers in biopsies from primary tumors or metastases; nevertheless, there is a lack of prospective trials assessing the role of these markers in the advanced setting, and misleading interpretations of retrospective results may lead to an inappropriate use of them.

In this review, our objective is to analyze the role of the expression of the proliferation marker $\mathrm{Ki}-67$ and review the relevant studies of chemotherapy in the digestive NETs with a view to identify specific subgroups of patients that derive the largest benefit from this therapeutic option.

\section{Role of KI-67 proliferation index in diges- tive NETs}

Most of the authors agree on that the indication of chemotherapy in digestive NETs should be based on the natural history of the disease, which acts as a necessary surrogate parameter of the biological behavior within this heterogeneous group of tumors. Candidates would be patients with advanced disease who have progressed in a short period of time of 3-12 months. However, hepatic and lymph node metastasis excision or locoregional techniques such as radiofrequency should be considered firstly whenever possible. Embolization or chemoembolization of the hepatic arteries may also be considered. In addition, in many cases, chemotherapy should not be considered a standard first-line systemic treatment; instead, biological therapies, such as somatostatin analogs (SAs) and interferon (IFN)- $\alpha$, would represent better options (Rougier \& Mitry 2000, Kaltsas et al. 2004, Oberg et al. 2004a). An alternative approach is to try to identify the pathological features to assist in characterizing subgroups with similar biological behavior that may lead to a better patient selection for chemotherapy treatments.

The combination of microscopic structural analysis and immunohistochemical detection of cell cycle-related antigens may distinguish the level of aggressiveness in NETs. There are several antibodies against different cell cycle proteins available in the market to quantify the proliferation by immunohistochemistry in formalin-fixed paraffin-embedded tissue, such as proliferation cell nuclear antigen (PCNA) and Ki-67. PCNA is a protein found in the nucleus that acts as a cofactor of DNA polymerase $\delta$ and increases the processivity of leading strand synthesis during DNA replication. The antibody against it recognizes a $36 \mathrm{kDa}$ protein that is present during the S phase (Lloyd 1998); however, in response to DNA damage, it is ubiquitinated and associated in the RAD6-dependent DNA repair pathway, so its detection will mark cell proliferation and DNA repair processes (Hoege et al. 2002). The Ki-67 protein is a proliferation antigen, which is present in G1, S, G2, and M phases of the cell cycle. Quiescent or resting cells in the G0 phase of the cell cycle do not express the Ki-67 antigen. Probably, the Ki-67 index is the best available marker of proliferation because it is not involved in DNA repair process as PCNA; MIB-1 monoclonal antibody is the most commonly used because it is readily available and compares well with other antibodies against $\mathrm{Ki}-67$ due to the high specificity of nuclear versus background staining (Lloyd 1998). In the majority of publications, Ki-67 proliferation index is assessed by scanning the stained slide to determine the area of maximum staining intensity. The number of positive cells in a total of 2000 or more tumor cells observed in ten or more representative high-power fields (HPF) is counted (Rindi et al. 2006).

There are several studies in PETs as well as in CTs that attempt to determine the role of PCNA and Ki-67 as predictors of biological behavior (Table 1).

Pelosi (Pelosi et al. 1992) showed correlation of expression between PCNA and Ki-67 indexes in resected PETs. They divided patients into three cohorts according to PCNA indexes that inversely correlated with mean survival, so that all those with PCNA $>5 \%$ had extrapancreatic extension of the disease and a shorter mean survival. A later study by the same group (Pelosi et al. 1996) analyzed several factors as predictors of survival and, although univariate analysis showed that Ki-67, PCNA, mitotic index, stage, and non-functioning type correlated with survival, multivariate analysis yielded only Ki-67 as an independent predictor using a cut-off value of $5 \%$. Another Italian group has reviewed 61 cases of non-functioning PETs; 17 clinicopathological variables and several immunohistochemical markers were investigated as candidate prognostic factors for metastasis and local invasion; several factors were significant in the univariate analysis, although $\mathrm{Ki}$ 67 assessed by MIB- 1 with a cut-off value of $2 \%$ was the most sensitive and specific. Ki-67 ( $\leq 2$ vs $>2 \%$ ) also correlated with survival, but it was not confirmed to be an independent factor in the multivariate analysis for 
Table 1 Studies assessing PCNA and Ki-67 in PETs and CTs

\begin{tabular}{|c|c|c|c|c|c|c|c|c|}
\hline Ref & $\begin{array}{c}\text { NET } \\
\text { subtype }\end{array}$ & $n$ & Fx/Non-Fx & Mx & $\begin{array}{l}\text { Lymph } \\
\text { node Mx }\end{array}$ & IHC & Cor OS & $\begin{array}{c}\text { Cut-off for OS } \\
(\%)\end{array}$ \\
\hline Pelosi et al. (1992) & PET & 35 & $17 / 18$ & - & - & $\begin{array}{l}\text { PCNA } \\
\text { Ki-67 }\end{array}$ & $\begin{array}{l}\downarrow \text { OS } \\
\text { NA }\end{array}$ & $>5$ \\
\hline Pelosi et al. (1996) & PET & 54 & $23 / 31$ & - & - & $\begin{array}{l}\text { Ki-67 } \\
\text { PCNA } \\
\text { PgRP }\end{array}$ & $\begin{array}{l}\downarrow \mathrm{OS} \\
\mathrm{NC} \\
\mathrm{NC}\end{array}$ & $>5$ \\
\hline La Rosa et al. (1996) & PET & 61 & $0 / 61$ & 34 & 12 & $\mathrm{Ki}-67$ & $\downarrow$ OS & $>2$ \\
\hline Gentil Perret et al. (1998) & PET & 35 & $14 / 21$ & 20 & - & Ki-67 & $\downarrow$ OS & $>4$ \\
\hline Clarke et al. (1997) & PET & 37 & $25 / 12$ & 21 & 13 & Ki-67 & $\downarrow$ OS & $>10$ \\
\hline Jorda et al. (2003) & PET & 39 & $20 / 19$ & 17 & 7 & Ki-67 & NA & \\
\hline Hochwald et al. (2002) & PET & 87 & $37 / 50$ & - & - & Ki-67 & $\downarrow$ OS & $>50 \mathrm{HPF}$ \\
\hline Rigaud et al. (2001) & PET & 32 & $0 / 32$ & 12 & 6 & Ki-67 & $\downarrow$ OS & $>2$ \\
\hline Chaudhry et al. (1992) & $\mathrm{PET} / \mathrm{CT}$ & 32 & - & - & - & Ki-67 & $\downarrow$ OS & $>0.3$ cells $/ \mathrm{mm}^{2}$ \\
\hline Kawahara et al. (2002) & CT & 41 & & 4 & & Ki-67 & $\mathrm{NC}$ & - \\
\hline Sokmensuer et al. (2001) & CT & 37 & - & - & - & $\begin{array}{l}\text { PCNA } \\
\text { Ki-67 }\end{array}$ & NC & - \\
\hline Canavese et al. (2001) & $\mathrm{PET} / \mathrm{CT}$ & 109 & $32 / 14$ & - & - & Ki-67 & NA & - \\
\hline
\end{tabular}

Fx, functioning; Non-Fx, non-functioning; IHC, immunohistochemistry; Mx, metastatic tumors; Cor OS, correlation with overall survival; NA, not assessed; NC, not correlated with overall survival; NET, neuroendocrine tumor; PET, pancreatic endocrine tumor; CT, carcinoid tumor; HPF, high power field.

survival, either (La Rosa et al. 1996). Median Ki-67 value among well-differentiated tumors was $6.4 \%$, which contrasts with a higher median value $(26.1 \%)$ in the poorly differentiated subgroup. Gentil Perret et al. (1998) has found correlation between $\mathrm{Ki}-67$ determined by MIB-1 and tumor size, histological differentiation, local invasion, and occurrence of metastasis in 35 patients with PETs who underwent surgical operation. Twenty of them had distant metastasis at the time of surgery. Stage III and IV patients according to the Klöppel classification had a mean Ki-67 value of 6.3 and $11.9 \%$ respectively. A receiver operator characteristic (ROC) curve constructed with data from this study showed that a proliferation index higher than $4 \%$ predicted for significant increase in the risk of death. In contrast, Clarke et al. (1997) established a cut-off value of $10 \%$ as a significant predictor of metastasis. Survival of patients whose tumors had a $\mathrm{Ki}-67$ value of $\geq 10 \%$ was significantly decreased compared with that of those whose tumors had a Ki-67 value of $<10 \%$ (19 vs 72 months). Mean Ki-67 was significantly higher in non-functioning than in functioning tumors $(20.9$ vs $5.1 \%, P=0.01)$ and in metastatic than in non-metastatic tumors (15.6 vs 3.3\%, $P=0.04$ ), and the logistic regression and proportional hazards model showed that Ki-67 was an independent significant predictor of metastasis and survival after adjusting for functional status and tumor size. Jorda et al. (2003) used a semiquantitative scoring system to explore its application for assessing the metastatic behavior of PET. All metastatic tumors expressed at least an intermediate $\mathrm{Ki}-67$-positive reaction $(5-25 \%)$; these results supporting the idea that low nuclear proliferative activity is unlikely to correlate with an advanced stage. The influence of Ki-67 on survival for patients with PETs, who have undergone curative resection at MSKCC $(n=87)$ for two decades, has also been reviewed (Hochwald et al. 2002). An MIB-1 value of more than 50 per $10 \mathrm{HPF}$ was associated with a significant worse disease-free and disease-specific survival on univariate analysis, but did not remain significant on multivariate analysis. More recently, a study performed on nonfunctioning PETs has evaluated several clinical, pathological (ploidy and Ki-67), and molecular factors (subgroups defined by allelotype) to predict survival (Rigaud et al. 2001). Only ploidy and Ki-67 remained significant in the multivariate analysis. Patients with $\mathrm{Ki}-67>2 \%$ had a relative risk of death of 11.7 when Cox's model was performed.

With regard to CTs, several authors (Chaudhry et al. 1992, Sokmensuer et al. 2001, Kawahara et al. 2002) have demonstrated a similar correlation between low $\mathrm{Ki}-67$ values and longer survival, although a clear cutoff value is not defined. In addition, one publication reported in the literature includes the analysis of both CTs and PETs (Canavese et al. 2001). This paper reports on the results of $\mathrm{Ki}-67$ along with other immunochemistry markers of 109 gastrointestinal NETs from the University of Parma. The authors reviewed the primary specimens of 46 PETs, 51 CTs and 12 poorly differentiated tumors. Malignancy was defined according to WHO criteria as evidence of invasion of surrounding tissues and/or metastasis to 
lymph nodes or distant sites. Mean Ki-67 value was found to be significantly different between benign and malignant tumors $(0.05$ vs $1.99 \%, P<0.0001)$. Poorly differentiated tumors were characterized by a very high Ki-67 index that was $>14 \%$ in all cases. Interestingly, midgut carcinoids presented low Ki-67 values, despite they met malignancy criteria (median $0.1 \%$ ).

In summary, these studies include patients affected by locoregional or advanced PETs, CTs, or both who have undergone surgery. They are retrospective and their common objective is to evaluate whether $\mathrm{Ki}-67$ proliferation index could be a prognostic factor of metastasis or survival. The evidence is convincing in that Ki-67 evaluation yields prognostic information of added value to the classical pathological report whenever it is performed in laboratories, where the test has been validated. In fact, in the last TNM staging proposal for foregut NETs Ki-67 is determined to asses the tumor grade and three groups are defined: $\leq 2 \%$, between 3 and $20 \%$, and $>20 \%$ (Rindi et al. 2006). However, there are several caveats to consider in the interpretation of the results from these studies. First, most of these studies have reviewed a single-institution experience with PETs or CTs that have been surgically resected (Pelosi et al. 1992, La Rosa et al. 1996, Clarke et al. 1997, Hochwald et al. 2002, Jorda et al. 2003). Secondly, patients were considered to have advanced disease if they presented with either locoregional lymph node or distant metastasis (La Rosa et al. 1996, Clarke et al. 1997, Hochwald et al. 2002, Jorda et al. 2003). Thirdly, prognostic factors are derived from the clinicopathological and immunohistochemical characteristics of the primary tumor and they are retrospectively correlated with patient outcome independently of the initial staging. Fourthly, only two studies includes both tumor primary types, PETs and CTs (Canavese et al. 2001, Panzuto et al. 2005), so comparison of Ki-67 prognostic value against primary site type or location in large series is lacking.

Based on the former studies that state the role of $\mathrm{Ki}-67$ as a prognostic factor, several authors and guidelines (Öberg 2002, Oberg et al. 2004b) have proposed that the $\mathrm{Ki}-67$ value could be a valid surrogate marker of biological behavior, and it could possibly substitute for clinical course surveillance to establish the indication of chemotherapy. Given the current evidence, it is sensible to hypothesize that tumors with high Ki-67 may respond better to chemotherapy, but this should be tested in prospective clinical studies that assess the role of $\mathrm{Ki}-67$ as a predictive factor of responsiveness to chemotherapy or other biological therapies. One limitation to this approach is the potential intratumoral heterogeneity and the differential expression between metastasis and primary tumors, when faced with a biopsy sample in a metastatic setting, which could jeopardize the clinical validation of Ki-67 as a predictive factor in this group of patients (Chaudhry et al. 1992), as it has been showed in other neoplasms (Dodd et al. 1997, Yasui et al. 2006).

\section{Chemotherapy in PETs}

PETs, also known as islet cell carcinomas, are rare neoplasms with an incidence rate of around five cases per million persons. The majority are non-functioning and within-functioning PETs; insulinoma and gastrinoma are the most common (Tomassetti et al. 2001).

There are a number of solid arguments that support the indication of chemotherapy as an effective treatment in PETs. First, it is well established that streptozocin (STZ) selectively targets pancreatic islet cells (Murray-Lyon et al. 1968), possibly through a transport system that is present on pancreatic $\beta$-cells and recognizes the glucose moiety of STZ (Kawada et al. 1987). Secondly, Ki-67 is, on average, higher in PETs than in CTs (Canavese et al. 2001), which may render them candidates for chemotherapy earlier in the treatment plan. High Ki-67 values may relate to other poor prognostic features such as higher tumor size (mean 5-6 cm), local extension, perineural or angioinvasion, and lymph node or distant metastasis (Clarke et al. 1997, Gentil Perret et al. 1998, Rindi et al. 1998, Pape et al. 2004). In addition, the majority of well-differentiated PETs are non-functioning, which in some reports have higher Ki-67 than functioning tumors (Clarke et al. 1997). Finally and most importantly, data from phase II and III trials show that PETs respond to a number of chemotherapeutic agents, and that combination regimens are better than monotherapy (Moertel et al. 1980, 1992).

Several cytotoxic agents have been evaluated in monotherapy (Table 2). In a phase II trial, doxorubicin (DOX) showed an response rate (RR) of $20 \%$ in 20 patients previously treated (Moertel et al. 1982). A phase II trial conducted by the ECOG tried to assess the efficacy of dacarbazine (DTIC; Ramanathan et al. 2001). The RR was $34 \%$ in 50 patients included, with a median survival of 19.3 months and a mild toxicity profile. High-dose paclitaxel with G-CSF support has been analyzed in a phase II trial (Ansell et al. 2001). Nine patients with advanced PETs were included showing no radiological and only one biochemical response. Therefore, taxanes are considered inactive for this group of tumors. Likewise, gemcitabine showed no radiological or biochemical responses among seven patients with PETs (Kulke et al. 2004b).

Well-designed and adequately sized prospective randomized trials that study the role of 
Table 2 Chemotherapy in PETs

\begin{tabular}{|c|c|c|c|c|}
\hline Author & Chemotherapy & Pts $(n)$ & $\mathbf{R R}(\%)$ & mOS (mo) \\
\hline \multirow[t]{2}{*}{ Moertel et al. (1980) } & $\mathrm{STZ}+5-\mathrm{FU}$ & 42 & 63 & 26 \\
\hline & STZ & 42 & 36 & 16.5 \\
\hline \multirow[t]{3}{*}{ Moertel et al. (1992) } & STZ+DOX & 36 & 69 & 26.4 \\
\hline & STX +5-FU & 33 & 45 & 16.8 \\
\hline & CLZ & 33 & 30 & 18 \\
\hline Ramanathan et al. (2001) & DTIC & 50 & 34 & 19 \\
\hline Ansell et al. (2001) & Paclitaxel & 10 & 10 & 13 \\
\hline Kulke et al. $(2004 b)$ & Gemcitabine & 7 & 0 & 11.5 \\
\hline Bukowski et al. (1992) & $\mathrm{CLZ}+5-\mathrm{FU}$ & 44 & 36 & 25 \\
\hline McCollum et al. (2004) & STZ+DOX & 16 & 6 & 20.2 \\
\hline Cheng \& Saltz (1999) & STZ+DOX & 16 & 6 & NR \\
\hline Kouvaraki et al. (2004) & $5-F U+S T Z+D O X$ & 84 & 39 & 37 \\
\hline Bajetta et al. (2002) & 5-FU + DTIC + epirubicin & 28 & 28.5 & NA \\
\hline Kulke et al. (2006a) & Temozolomide + thalidomide & 11 & 45 & NA \\
\hline Kulke et al. (2006b) & Temozolomide + bevacizumab & 18 & 24 & NA \\
\hline
\end{tabular}

PET, pancreatic endocrine tumor; Pts, patients; RR, response rate; mOS, median overall survival; Ref, reference; NA, not assessed; $\mathrm{NR}$, not reached.

polychemotherapy are lacking. Randomized controlled trials conducted in the Mayo Clinic during the 1970s and 1980s (Table 2) produced much of the evidence of what is still considered the standard of care. A total of 103 patients were randomized to receive STZ and 5-fluorouracil (5-FU) or STZ in a phase III trial conducted by Moertel et al. (1980). The combination treatment was better than the single-agent arm in terms of response, 63 and $36 \%$ respectively, and also yielded a survival advantage, although this did not reach statistical significance. These results led the ECOG to design a new phase III trial to compare STZ and DOX versus STZ and 5-FU versus chlorozotocin (CLZ) in monotherapy (Moertel et al. 1992). The DOX combination group showed a significant increase in RR (69 vs 45 vs $30 \%$ respectively). Additionally, this benefit was also translated into a statistically significant increase in time to progression (TTP; 18 vs 14 vs 17 months) and median overall survival (mOS; 26.4 vs 16.8 vs 18 months). The SWOG group has published its experience on 47 patients treated with CLZ and 5-FU (Bukowski et al. 1992). This combination showed an RR of $36 \%$ and an mOS of 25 months. Thirteen patients developed renal toxicity secondary to CLZ, which explains the limited use of this agent in subsequent trials.

Subsequent retrospective reports of the use of the STZ plus DOX combination in two different institutions have been published. Both the Dana Farber Cancer Institute (McCollum et al. 2004) and the Memorial Sloan Kettering Cancer Center (Cheng \& Saltz 1999) have reported their experience, with an RR of $6 \%$, much lower than the activity reported earlier. This disparity of results may be explained by the methodology used to assess response in the old studies, where clinical measurement of hepatomegaly on physical examination, radionuclide liver-spleen scanning, and biological response were considered for response evaluation. It may also be partially explained by tumor heterogeneity in terms of biological behavior and responsiveness to chemotherapy. Another potential explanation is the fact that in the last decade biological therapies have been progressively introduced as first- or second-line treatment, starting chemotherapy only after the progression of those therapies (Vilar et al. 2005).

In an intent to improve the efficacy results with doublets obtained in these two modern series, and as it has been done in other malignancies, triple cytotoxic combinations with 5-FU, DOX, and STZ have also been retrospectively reviewed in 84 patients treated at MD Anderson Cancer Center (Kouvaraki et al. 2004). The majority of patients had non-functioning $(n=64)$ and metastatic tumors $(n=76)$, although some locally advanced were also included in the analysis $(n=8)$. The overall RR was $39 \%$, and $50 \%$ achieved a stable disease (SD). In terms of response, no differences were observed between locally advanced and metastatic tumors. Median progression-free survival (PFS) and PFS at 2 years were 18 months and $41 \%$ respectively; mOS and overall survival (OS) at 2 years were 37 months and $74 \%$ respectively. Multivariate analysis showed that replacement of more than $75 \%$ of the liver was independently associated with inferior PFS.

Due to the disagreement concerning the therapeutic effect of the classical chemotherapy regimens used in PETs, several groups have studied other combinations. The Italian Trials Medical Oncology group evaluated the efficacy of a combination of 5-FU, DTIC, and epirubicin and its dose intensification (Bajetta et al. 2002). Their 
pooled data published in 2002 included 28 patients with pancreatic tumors. Among these, one complete response (CR), seven partial responses (PR), and eight stabilizations were observed. The ECOG performed a phase II trial in advanced NETs to assess the efficacy of the combination of temozolomide and thalidomide (Kulke et al. 2006a). Temozolomide is an oral prodrug of DTIC with better bioavailability profile. Thalidomide is postulated to have an antiangiogenic effect and, as NETs are characterized by abundant vasculature, therapies related to these pathways may be especially relevant. Eleven patients affected by PET were treated; 1 (CR) and 4 (PR) were observed in this group. This regimen seems to be especially active in pancreatic tumors. Another phase II study of the combination of temozolomide and bevacizumab was reported (Kulke et al. 2006c) during the 2006 ASCO Meeting. The authors enrolled 18 patients with PETs who had received several prior treatment lines. Four patients experienced PR (24\%) and $12 \mathrm{SD}(70 \%)$, thus this combination has shown promising activity that has not been confirmed in patients with CT $(n=12,0$ PR and $11 \mathrm{SD})$. Experience with temozolomide and capecitabine has been reviewed retrospectively in 17 patients with PETs. One patient achieved a (CR), and nine a (PR) with a median duration of partial response of 284 days; therefore, this combination has a promising activity that should be evaluated in further studies (Isacoff et al. 2006).

One old study by Moertel et al. (1994) analyzes the combination of chemotherapy with a locoregional treatment. A total of 46 patients with PETs and liver metastasis were treated with hepatic artery occlusion followed by a non-randomized investigator's choice of sequential chemotherapy or observation. Arterial occlusion was done by surgical ligation or by catheterization and embolization. The chemotherapy regimen included DOX and DTIC and, 4 weeks later, STZ and 5-FU, and cycles were administered until the patient had reached the stabilization of the disease or at a maximum tumor regression or had reached the accumulated dose limit in the case of DOX or had experienced intolerable toxicity. Twenty-nine patients were given the combined treatment and 17 with hepatic artery occlusion alone. The regression rate and median TTP were $78 \%$ and 20 months, and $43 \%$ and 3.6 months respectively. Despite the limitations and potential bias of this study, due to its non-randomized design, its results suggest that locoregional treatment of liver metastasis may enhance the efficacy of subsequent chemotherapy and supports the rationale for exploring combined sequential treatments.

\section{Chemotherapy in gastrointestinal CTs}

CTs are the most frequent NETs. The incidence rate in the digestive system is unknown because the true incidence is underestimated. However, the latest reports estimate it at around four cases per 100000 population per year (Modlin et al. 2003).

Several drugs have been evaluated as single-agent regimens (Table 3). A phase II trial (Moertel et al. 1986) tested the efficacy of cisplatin (CDDP). Fifteen

Table 3 Chemotherapy in CTs

\begin{tabular}{|c|c|c|c|c|}
\hline Author & Chemotherapy & Pts $(n)$ & $\mathbf{R R}(\%)$ & mos (mo) \\
\hline \multirow[t]{2}{*}{ Engstrom et al. (1984) } & $5-F U+S T Z$ & 86 & 22 & 16 \\
\hline & DOX & 86 & 21 & 12 \\
\hline Moertel \& Hanley (1979) & $5-\mathrm{FU}$ & 19 & 26 & \\
\hline Moertel et al. (1986) & CDDP & 15 & 7 & NA \\
\hline \multirow[t]{2}{*}{ Moertel \& Hanley (1979) } & $5-F U+S T Z$ & 118 & 33 & ND \\
\hline & $\mathrm{CICLOP}+\mathrm{STZ}$ & & 26 & \\
\hline \multirow[t]{2}{*}{ Oberg et al. (1987) } & STZ & 7 & 3.2 & 7.5 \\
\hline & $\mathrm{STZ}+5-\mathrm{FU}$ & 24 & 6.4 & 18 \\
\hline Bukowski et al. (1994) & DTIC & 63 & 16 & 20 \\
\hline \multirow[t]{2}{*}{ Bukowski et al. (1987) } & $5-\mathrm{FU}+\mathrm{STZ}+\mathrm{CICLOP}+\mathrm{DOX}$ & 56 & 31 & ND \\
\hline & $5-\mathrm{FU}+\mathrm{STZ}+\mathrm{CICLOP}$ & 9 & 22 & \\
\hline Kulke et al. (2006a) & Temozolomide + thalidomide & 14 & 7 & NA \\
\hline Kulke et al. (2004b) & Gemcitabine & 9 & 0 & ND \\
\hline Kulke et al. (2004a) & Docetaxel & 21 & 0 & 24 \\
\hline Ansell et al. (2001) & Paclitaxel & 14 & 7 & ND \\
\hline Bajetta et al. (1998) & 5-FU+DTIC + epirubicin & 6 & 17 & NA \\
\hline Ollivier et al. (1998) & $5-\mathrm{FU}+$ leucovorin + DTIC & 9 & 11 & NA \\
\hline \multirow[t]{2}{*}{ Sun et al. (2005) } & $5-F U+S T Z$ & 249 & 16 & 24.3 \\
\hline & $5-F U+D O X$ & & 15.9 & 15.7 \\
\hline
\end{tabular}

CT, carcinoid tumor; Pts, patients; RR, response rate; mOS, median overall survival; Ref, reference; NA, not assessed; ND, not determined. 
patients were treated showing only one (PR). STZ has shown very little activity in monotherapy (Oberg et al. 1987). DTIC (Bukowski et al. 1994) was evaluated in a phase II trial with 63 patients, exhibiting an RR of $16 \%$. Two studies assessed the efficacy of gemcitabine (Kulke et al. 2004b) and docetaxel (Kulke et al. 2004a) in monotherapy; both treatments were considered inactive because neither of them induced any radiological response. Likewise, 14 patients were enrolled in a trial with high-dose paclitaxel (Ansell et al. 2001) in the Mayo Clinic showing minimum clinical activity despite severe toxicities, mainly hematological. In summary, single-agent chemotherapy should not be considered as a therapeutic option.

Despite the poor results of the former trials, several phase II trials have explored the role of combined chemotherapy. The most frequently assessed combination is STZ plus 5-FU. Moertel \& Hanley (1979) randomized 118 patients to receive STZ plus 5FU or STZ plus cyclophosphamide. They reported an RR of 33 and 26\% respectively. Among 11 patients who received crossover therapy with 5-FU alone, there were two responses. EST 5275 (Engstrom et al. 1984) randomized patients with CT to receive STZ plus 5-FU or single-agent DOX showing an RR of $22 \%$ for the combination treatment and $21 \%$ for DOX. Oberg et al. (1987) observed three objective responses (10\%) in 31 patients treated with this combination. This lower RR observed by the Uppsala group points in the same direction about the disparity of results from initial old randomized trials and subsequent reports that have been discussed in PETs.

The two studies from the ECOG stimulated the design of a phase III trial (study E1281; Sun et al. 2005) which randomized 163 patients to receive 5-FU plus DOX or STZ plus 5-FU. Results in terms of RR (15.9 vs $16 \%, P=0.82)$ or PFS (4.5 vs 5.3 months, $P=$ 0.17 ) did not show any differences; however, STZ plus 5-FU was superior to 5-FU plus DOX in mOS (24.3 vs 15.7 months, $P=0.02$ ). The authors concluded that for selected patients the combination of STZ and fluoropyrimidines would be an appropriate option if systemic chemotherapy is considered.

In the Cleveland Clinic Foundation (Bukowski et al. 1987), 65 patients were randomized to 5-FU plus cyclophosphamide plus STZ with or without DOX showing an RR of 31 and $22 \%$ respectively. Another combination chemotherapy including 5-FU plus DTIC plus epirubicin (Bajetta et al. 1998) has been evaluated by an Italian collaborative group. In this trial, only two responses $(10 \%)$ out of 20 patients were observed. In a more recent study, the same regimen at intensified doses slightly increased the response to $17 \%$ with a remarkable increase in toxicity (Bajetta et al. 2002). Another combination with DTIC plus 5-FU and leucovorin (Ollivier et al. 1998) had poor results, with only one (PR) out of nine CTs treated. A recently published multi-institutional phase II trial has evaluated the combination of temozolomide and thalidomide (Kulke et al. 2006a) in patients with advanced NETs of several origins; among 15 patients with CT, there was only one response. Therefore, this combination does not seem to be active for patients with CT, either.

The main prognostic factors that have correlated with better response to chemotherapy are a good performance status and age under 60 years.

None of the regimens analyzed has demonstrated enough efficacy to consider chemotherapy upfront in the systemic treatment in patients with advanced CT. Hence, other forms of systemic antitumoral treatment based on the presence of somatostatin receptors and other molecular characteristics as expression of vascular endothelial growth factor in CT are being used and explored. One of the options that has been explored is the combination of biological agents with target therapies and, as a consequence, a planned phase III study will randomize patients into two different treatment arms, octreotide plus bevacizumab versus octreotide plus IFN. Other strategy is the use of radiolabeled SAs which has shown interesting results in terms of tumor responses associated with low rates of toxicity in heavily pretreated patients (Kwekkeboom et al. 2005).

Locoregional treatments also show higher antitumoral activity in terms of RR than systemic treatments, as it has been observed in a prospective study comparing chemotherapy with STZ plus 5-FU, transcatheter arterial embolization, and SAs in digestive NETs (Skinazi et al. 1996, Roche et al. 2003). In addition, Moertel et al. (1994) analyzed the combination of hepatic arterial occlusion and sequential chemotherapy. Sixty-five patients affected by advanced CTs with liver metastasis were included. Twenty-three patients were treated with hepatic arterial occlusion and 42 patients with the combined modality treatment that was stated in the welldifferentiated PET section. The RR and median duration of response were $56 \%$ and 6.6 months, and $75 \%$ and 19.8 months respectively. However, a recent report has yielded conflicting results, where a sequential effect between embolization and chemotherapy has not been confirmed. In this study, the OS of patients who received postembolization chemotherapy was 38 months, whereas in the group that did not 
receive chemotherapy it was 44 months (Strosberg et al. 2006).

Multiple studies have demonstrated that locoregional techniques in CT render a higher benefit than chemotherapy in terms of RR; but despite this evidence, they are generally used as an alternative treatment after failure to systemic therapies rather than upfront which would seem a sensible approach. Studies of sequential or concomitant combination of both strategies in first-line treatment are needed to decide what the best sequence is, in order to improve the outcome in this subgroup of digestive NETs.

\section{Poorly differentiated gastroenteropan- creatic NETs or anaplastic gastroentero- pancreatic tumors}

The aggressiveness of poorly differentiated gastroenteropancreatic NETs or anaplastic tumors (also referred as large or small cell tumors) is similar to small cell lung cancer, having a median OS of 7 months without treatment.

Moertel et al initially reported an RR of $67 \%$ (CR $17 \%$, PR $50 \%$, and SD 33\%) with the combination of CDDP and etoposide (VP-16) in 18 patients with poorly differentiated tumors of gastrointestinal and pancreatic origin. The RR for patients with well-differentiated tumors was $7 \%(n=27$, PR $7 \%)$, emphasizing the fact that this regimen is less effective for this group (Moertel et al. 1991). Mitry et al. (1999) observed a RR for poorly $(n=41)$ and well-differentiated $(n=11)$ NETs of 41.5 and $9 \%$ respectively, thus confirming the results obtained by the Mayo Clinic group.

In contrast, another study with this regimen in second- and third-line treatments does not confirm the relationship between the grade of differentiation and RR. A possible explanation is that patients with welldifferentiated tumors included in these trials had a rapidly progressing clinical course and some of them were atypical lung carcinoids, which are more responsive to cisplatin-based chemotherapy (Fjallskog et al. 2001).

A recent phase II trial of the combination of paclitaxel, carboplatin, and VP-16 has been reported by Hainsworth et al. (2006). Seventy-eight patients with histologically proven poorly differentiated neuroendocrine carcinoma with a variety of known primary sites with the exception of lung were included. A majority of them were from an unknown primary site, and nine patients had primaries located in the colon, rectum, esophagus, and stomach. Fifty-three percent showed major responses with a complete RR of $15 \%$ and median OS of 14.5 months. PFS and OS were not significantly different when unknown and known primary sites were compared. Nevertheless, toxicity was important, grade $3 / 4$ neutropenia being present in $82 \%$ of patients and three patients died as a consequence of neutropenic sepsis. This remarkable toxicity rate counterbalances the efficacy results of this combination, thus CDDP plus VP-16 regimens continue being the standard treatment in this population. Kulke et al. (2006b) have reported the efficacy of the combination regimen of CDDP and irinotecan among 18 patients with advanced NETs. Only one radiological response was observed among four patients with poorly differentiated NETs and no radiological responses were observed in 14 patients with well-differentiated tumors. Efficacy of the oxaliplatin-based regimen FOLFOX-4 in 16 patients ( 8 low-grade and 8 high-grade) has been reported at the 2006 ASCO meeting by Wiedenmann et al. SD was observed in $62.5 \%$ patients, despite of the fact that the majority of patients $(n=13)$ had a Ki-67 value $\geq 10 \%$ (Pape et al. 2006), suggesting that this regimen may be an option in tumors with highly aggressive potential.

\section{Conclusions}

Chemotherapy has been used for the management of metastatic digestive NETs for the last three decades, but a clear definition of its indications is lacking. Recently, multiple clinicopathological variables have been developed to identify prognostic subgroups, but studies validating its predictive value of responsiveness to chemotherapy are also lacking. Vascular microinvasion and Ki-67 proliferative index are the most sensitive and specific factors for invasivity. High proliferating tumors that have a Ki-67 index higher than $10 \%$ are usually larger than $2 \mathrm{~cm}$, have extensive angioinvasion, and show a great potential to develop metastatic disease. In addition, Ki-67 is probably an independent prognostic factor for survival in both locoregional and advanced disease settings, and some authors argue that it could be a valid surrogate marker of biological behavior in order to establish the indication of chemotherapy. A possible therapeutic algorithm (Fig. 1) would favor chemotherapy as first choice in tumors with $\mathrm{Ki}-67>10 \%$ and as second or further - if at all indicated - line of treatment after failure of biotherapy and other forms of treatment in low proliferating tumors with Ki-67 lower than 5\% (Öberg 2002), but this approach needs to be validated in prospective trials. Furthermore, validation of Ki-67 and other clinical and pathological prognostic factors is urgently needed for better patient stratification in future clinical trials. This approach will help 


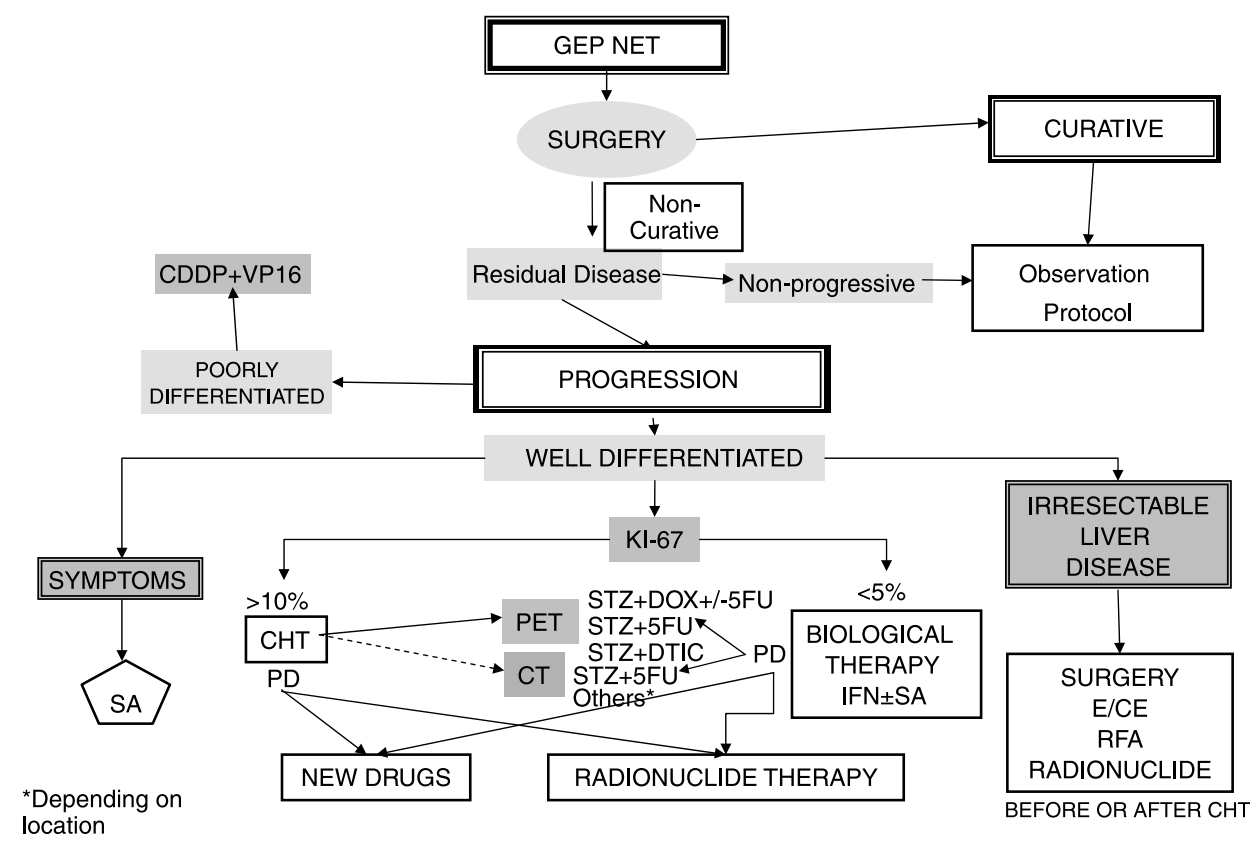

Figure 1 Suggested algorithm for treatment of gastroenteropancreatic neuroendocrine tumors. SA, somatostatin analogs; IFN, interferon; CHT, chemotherapy; PD, progresive disease; RFA, radiofrequency; E, embolization; CE, chemoembolization; PET, pancreatic endocrine tumors; CT, carcinoid tumors; gep, GEP, gastroenteropancreatic; NET, neuroendocrine tumors; STZ, streptozocin; DOX, doxorubicin; DTIC, dacarbazine; 5-FU, 5-fluorouracil; PD, progressive disease.

identifying which patient subgroups obtain the largest therapeutic benefit from the different old and new treatments potentially available in NETs. In the meantime, Ki-67 should only be considered a prognostic factor and a valid surrogate of biological behavior that could guide therapeutic decisions, but its predictive value of responsiveness to treatment is unknown.

On the other hand, PETs are remarkably more responsive to chemotherapy than CTs. Single-agent chemotherapy has been evaluated in multiple trials. STZ, DOX, 5-FU, and DTIC or its pro-drug temozolomide are the most active agents with an RR in the range of $15-35 \%$ in PETs and $5-15 \%$ in CTs. Polychemotherapy obtains higher RR (probably in the range of 40\%) and longer OS than monotherapy in PETs. Combinations of STZ plus 5-FU or DOX or both are the standard of care in PETs. The combination of CDDP plus VP-16 is not questioned as the mainstay of the treatment of poorly differentiated digestive tumors. Chemotherapy is not considered as a standard frontline treatment for CTs and the combination of STZ and 5-FU may be an option only after other forms of treatment have failed.

In light of the results achieved with the current treatment approaches, a logical therapeutic and investigational approach should include sequential treatments with aggressive locoregional strategies and systemic treatment, a better candidate selection based on predictive validated clinical, pathological, and molecular factors, development of new targeted therapies, and the combination of these with chemotherapy or with other targeted therapies. These strategies should derive in the continuous progress on survival improvements of the patients affected by NETs.

\section{Acknowledgements}

The authors declare that there is no conflict of interest that would prejudice the impartiality of this scientific work

\section{References}

Ansell SM, Pitot HC, Burch PA, Kvols LK, Mahoney MR \& Rubin J 2001 A phase II study of high-dose paclitaxel in patients with advanced neuroendocrine tumors. Cancer 91 1543-1548.

Bajetta E, Rimassa L, Carnaghi C, Seregni E, Ferrari L, Di Bartolomeo M, Regalia E, Cassata A, Procopio G \& Mariani L 1998 5-Fluorouracil, dacarbazine, and epirubicin in the treatment of patients with neuroendocrine tumors. Cancer 83 372-378. 
Bajetta E, Ferrari L, Procopio G, Catena L, Ferrario E, Martinetti A, Di Bartolomeo M, Buzzoni R, Celio L, Vitali M et al. 2002 Efficacy of a chemotherapy combination for the treatment of metastatic neuroendocrine tumours. Annals of Oncology 13 614-621.

Bukowski RM, Johnson KG, Peterson RF, Stephens RL, Rivkin SE, Neilan B \& Costanzi JH 1987 A phase II trial of combination chemotherapy in patients with metastatic carcinoid tumors. A Southwest Oncology Group Study. Cancer 60 2891-2895.

Bukowski RM, Tangen C, Lee R, Macdonald JS, Einstein AB Jr, Peterson R \& Fleming TR 1992 Phase II trial of chlorozotocin and fluorouracil in islet cell carcinoma: a Southwest Oncology Group study. Journal of Clinical Oncology 10 1914-1918.

Bukowski RM, Tangen CM, Peterson RF, Taylor SA, Rinehart JJ, Eyre HJ, Rivkin SE, Fleming TR \& Macdonald JS 1994 Phase II trial of dimethyltriazenoimidazole carboxamide in patients with metastatic carcinoid. A Southwest Oncology Group study. Cancer 73 1505-1508.

Canavese G, Azzoni C, Pizzi S, Corleto VD, Pasquali C, Davoli C, Crafa P, Delle Fave G \& Bordi C 2001 p27: a potential main inhibitor of cell proliferation in digestive endocrine tumors but not a marker of benign behavior.

Human Pathology 32 1094-1101.

Chaudhry A, Oberg K \& Wilander E 1992 A study of biological behavior based on the expression of a proliferating antigen in neuroendocrine tumors of the digestive system. Tumour Biology 13 27-35.

Cheng PN \& Saltz LB 1999 Failure to confirm major objective antitumor activity for streptozocin and doxorubicin in the treatment of patients with advanced islet cell carcinoma. Cancer 86 944-948.

Clarke MR, Baker EE, Weyant RJ, Hill L \& Carty SE 1997 Proliferative activity in pancreatic endocrine tumors: association with function, metastases, and survival. Endocrine Pathology 8 181-187.

Dodd LG, Kerns BJ, Dodge RK \& Layfield LJ 1997 Intratumoral heterogeneity in primary breast carcinoma: Study of Concurrent Parameters. Journal of Surgical Oncology 64 280-288.

Engstrom PF, Lavin PT, Moertel CG, Folsch E \& Douglass HO Jr 1984 Streptozocin plus fluorouracil versus doxorubicin therapy for metastatic carcinoid tumor. Journal of Clinical Oncology 2 1255-1259.

Fjallskog ML, Granberg DP, Welin SL, Eriksson C, Oberg KE, Janson ET \& Eriksson BK 2001 Treatment with cisplatin and etoposide in patients with neuroendocrine tumors. Cancer 92 1101-1107.

Gentil Perret A, Mosnier JF, Buono JP, Berthelot P, Chipponi J, Balique JG, Cuilleret J, Dechelotte P \& Boucheron S 1998 The relationship between MIB-1 proliferation index and outcome in pancreatic neuroendocrine tumors. American Journal of Clinical Pathology 109 286-293.

Hainsworth JD, Spigel DR, Litchy S \& Greco FA 2006 Phase II trial of paclitaxel, carboplatin, and etoposide in advanced poorly differentiated neuroendocrine carcinoma: a Minnie Pearl Cancer Research Network Study. Journal of Clinical Oncology 24 3548-3554.

Hochwald SN, Zee S, Conlon KC, Colleoni R, Louie O, Brennan MF \& Klimstra DS 2002 Prognostic factors in pancreatic endocrine neoplasms: an analysis of 136 cases with a proposal for low-grade and intermediate-grade groups. Journal of Clinical Oncology 20 2633-2642.

Hoege C, Pfander B, Moldovan G-L, Pyrowolakis G \& Jentsch S 2002 RAD6-dependent DNA repair is linked to modification of PCNA by ubiquitin and SUMO. Nature 419 135-141.

Isacoff WH, Moss RA, Pecora AL \& Fine RL 2006 Temozolomide/capecitabine therapy for metastatic neuroendocrine tumors of the pancreas. A retrospective review. Journal of Clinical Oncology 2414023.

Jensen RT \& Norton JA 1995 Endocrine tumors of the pancreas. In Textbook of Gastroenterology, pp 21312166. Eds T Yamada, BH Alpers \& C Owyang. Philadelphia: Lippincot.

Jorda M, Ghorab Z, Fernandez G, Nassiri M, Hanly A \& Nadji M 2003 Low nuclear proliferative activity is associated with nonmetastatic islet cell tumors. Archives of Pathology and Laboratory Medicine 127 196-199.

Kaltsas GA, Besser GM \& Grossman AB 2004 The diagnosis and medical management of advanced neuroendocrine tumors. Endocrine Reviews 25 458-511.

Kawada J, Okita M, Nishida M, Yoshimura Y, Toyooka K \& Kubota S 1987 Protective effect of 4,6-O-ethylidene glucose against the cytotoxicity of streptozotocin in pancreatic beta cells in vivo: indirect evidence for the presence of a glucose transporter in beta cells. Journal of Endocrinology 112 375-378.

Kawahara M, Kammori M, Kanauchi H, Noguchi C, Kuramoto S, Kaminishi M, Endo H \& Takubo K 2002 Immunohistochemical prognostic indicators of gastrointestinal carcinoid tumours. European Journal of Surgical Oncology 28 140-146.

Kloppel G, Perren A \& Heitz PU 2004 The gastroenteropancreatic neuroendocrine cell system and its tumors: the WHO classification. Annals of the New York Academy of Sciences 1014 13-27.

Kouvaraki MA, Ajani JA, Hoff P, Wolff R, Evans DB, Lozano R \& Yao JC 2004 Fluorouracil, doxorubicin, and streptozocin in the treatment of patients with locally advanced and metastatic pancreatic endocrine carcinomas. Journal of Clinical Oncology 22 4762-4771.

Kulke MH, Kim H, Stuart K, Clark JW, Ryan DP, Vincitore M, Mayer RJ \& Fuchs CS 2004a A phase II study of docetaxel in patients with metastatic carcinoid tumors. Cancer Investigation 22 353-359.

Kulke MH, Kim H, Clark JW, Enzinger PC, Lynch TJ, Morgan JA, Vincitore M, Michelini A \& Fuchs CS $2004 b$ A phase II trial of gemcitabine for metastatic neuroendocrine tumors. Cancer 101 934-939.

Kulke MH, Stuart K, Enzinger PC, Ryan DP, Clark JW, Muzikansky A, Vincitore M, Michelini A \& Fuchs CS 
$2006 a$ Phase II study of temozolomide and thalidomide in patients with metastatic neuroendocrine tumors.

Journal of Clinical Oncology 24 401-406.

Kulke MH, Wu B, Ryan DP, Enzinger PC, Zhu AX, Clark

JW, Earle CC, Michelini A \& Fuchs CS 2006 $b$ A phase II trial of irinotecan and cisplatin in patients with metastatic neuroendocrine tumors. Digestive Diseases and Sciences 51 1033-1038.

Kulke MH, Stuart K, Earle CC, Bhargava P, Clark JW, Enzinger PC, Meyerhardt J, Attawia M, Lawrence C \& Fuchs CS 2006c A phase II study of temozolomide and bevacizumab in patients with advanced neuroendocrine tumors. Journal of Clinical Oncology 244044.

Kwekkeboom DJ, Teunissen JJ, Bakker WH, Kooij PP, de Herder WW, Feelders RA, van Eijck CH, Esser JP, Kam BL \& Krenning EP 2005 Radiolabeled somatostatin analog $\left[{ }^{177} \mathrm{Lu}\right.$-DOTA0,Tyr3] octreotate in patients with endocrine gastroenteropancreatic tumors. Journal of Clinical Oncology 23 2754-2762.

Lloyd RV 1998 Utility of Ki-67 as a prognostic marker in pancreatic endocrine neoplasms. American Journal of Clinical Pathology 109 245-247.

McCollum AD, Kulke MH, Ryan DP, Clark JW, Shulman LN, Mayer RJ, Bartel S \& Fuchs CS 2004 Lack of efficacy of streptozocin and doxorubicin in patients with advanced pancreatic endocrine tumors. American Journal of Clinical Oncology 27 485-488.

Mitry E, Baudin E, Ducreux M, Sabourin JC, Rufie P, Aparicio T, Lasser P, Elias D, Duvillard P, Schlumberger M et al. 1999 Treatment of poorly differentiated neuroendocrine tumours with etoposide and cisplatin. British Journal of Cancer 81 1351-1355.

Modlin IM, Lye KD \& Kidd M 2003 A 5-decade analysis of 13715 carcinoid tumors. Cancer 97 934-959.

Moertel CG \& Hanley JA 1979 Combination chemotherapy trials in metastatic carcinoid tumor and the malignant carcinoid syndrome. Cancer Clinical Trials 2 327-334.

Moertel CG, Hanley JA \& Johnson LA 1980 Streptozocin alone compared with streptozocin plus fluorouracil in the treatment of advanced islet-cell carcinoma. New England Journal of Medicine 303 1189-1194.

Moertel CG, Lavin PT \& Hahn RG 1982 Phase II trial of doxorubicin therapy for advanced islet cell carcinoma. Cancer Treatment Reports 66 1567-1569.

Moertel CG, Rubin J \& O'Connell MJ 1986 Phase II study of cisplatin therapy in patients with metastatic carcinoid tumor and the malignant carcinoid syndrome. Cancer Treatment Reports 70 1459-1460.

Moertel CG, Kvols LK, O’Connell MJ \& Rubin J 1991 Treatment of neuroendocrine carcinomas with combined etoposide and cisplatin. Evidence of major therapeutic activity in the anaplastic variants of these neoplasms. Cancer 68 227-232.

Moertel CG, Lefkopoulo M, Lipsitz S, Hahn RG \& Klaassen D 1992 Streptozocin-doxorubicin, streptozocin- fluorouracil or chlorozotocin in the treatment of advanced islet-cell carcinoma. New England Journal of Medicine 326 519-523.

Moertel CG, Johnson CM, McKusick MA, Martin JK Jr, Nagorney DM, Kvols LK, Rubin J \& Kunselman S 1994 The management of patients with advanced carcinoid tumors and islet cell carcinomas. Annals of Internal Medicine 120 302-309.

Murray-Lyon IM, Eddleston AL, Williams R, Brown M, Hogbin BM, Bennett A, Edwards JC \& Taylor KW 1968 Treatment of multiple-hormone-producing malignant islet-cell tumour with streptozotocin. Lancet $\mathbf{2}$ 895-898.

Öberg K 2002 Management of neuroendocrine tumors. In ASCO Educational Book, pp 401-405.

Oberg K, Norheim I, Lundqvist G \& Wide L 1987 Cytotoxic treatment in patients with malignant carcinoid tumors. Response to streptozocin-alone or in combination with 5-FU. Acta Oncologica 26 429-432.

Oberg K, Kvols L, Caplin M, Delle Fave G, de Herder W, Rindi G, Ruszniewski P, Woltering EA \& Wiedenmann B $2004 a$ Consensus report on the use of somatostatin analogs for the management of neuroendocrine tumors of the gastroenteropancreatic system. Annals of Oncology 15 966-973.

Oberg K, Astrup L, Eriksson B, Falkmer SE, Falkmer UG, Gustafsen J, Haglund C, Knigge U, Vatn MH \& Valimaki M $2004 b$ Guidelines for the management of gastroenteropancreatic neuroendocrine tumours (including bronchopulmonary and thymic neoplasms). Part I-general overview. Acta Oncologica 43 617-625.

Ollivier S, Fonck M, Becouarn Y \& Brunet R 1998 Dacarbazine, fluorouracil, and leucovorin in patients with advanced neuroendocrine tumors: a phase II trial. American Journal of Clinical Oncology 21 237-240.

Panzuto F, Nasoni S, Falconi M, Corleto VD, Capurso G, Cassetta S, Di Fonzo M, Tornatore V, Milione M, Angeletti S, Cattaruzza MS, Ziparo V, Bordi C, Pederzoli P \& Delle Fave G 2005 Prognostic factors and survival in endocrine tumor patients: comparison between gastrointestinal and pancreatic localization. Endocrine-Related Cancer 12 1083-1092.

Pape UF, Bohmig M, Berndt U, Tiling N, Wiedenmann B \& Plockinger U 2004 Survival and clinical outcome of patients with neuroendocrine tumors of the gastroenteropancreatic tract in a german referral center. Annals of the New York Academy of Sciences 1014 222-233.

Pape U, Tiling N, Bartel C, Plockinger U \& Wiedenmann B 2006 Oxaliplatin plus 5-fluorouracil/folinic acid as palliative treatment for progressive malignant gastrointestinal neuroendocrine carcinomas. Journal of Clinical Oncology 2414074.

Pelosi G, Zamboni G, Doglioni C, Rodella S, Bresaola E, Iacono C, Serio G, Iannucci A \& Scarpa A 1992 Immunodetection of proliferating cell nuclear antigen assesses the growth fraction and predicts 
malignancy in endocrine tumors of the pancreas.

American Journal of Surgical Pathology 16 1215-1225.

Pelosi G, Bresaola E, Bogina G, Pasini F, Rodella S, Castelli P, Iacono C, Serio G \& Zamboni G 1996 Endocrine tumors of the pancreas: Ki-67 immunoreactivity on paraffin sections is an independent predictor for malignancy: a comparative study with proliferating-cell nuclear antigen and progesterone receptor protein immunostaining, mitotic index, and other clinicopathologic variables. Human Pathology 27 1124-1134.

Ramanathan RK, Cnaan A, Hahn RG, Carbone PP \& Haller DG 2001 Phase II trial of dacarbazine (DTIC) in advanced pancreatic islet cell carcinoma. Study of the Eastern Cooperative Oncology Group-E6282. Annals of Oncology 12 1139-1143.

Rigaud G, Missiaglia E, Moore PS, Zamboni G, Falconi M, Talamini G, Pesci A, Baron A, Lissandrini D, Rindi G et al. 2001 High resolution allelotype of nonfunctional pancreatic endocrine tumors: identification of two molecular subgroups with clinical implications. Cancer Research 61 285-292.

Rindi G, Capella C \& Solcia E 1998 Cell biology, clinicopathological profile, and classification of gastroenteropancreatic endocrine tumors. Journal of Molecular Medicine 76 413-420.

Rindi G, Kloppel G, Alhman H, Caplin M, Couvelard A, de Herder WW, Erikssson B, Falchetti A, Falconi M, Komminoth P et al. 2006 TNM staging of foregut (neuro)endocrine tumors: a consensus proposal including a grading system. Virchows Archiv 449 395-401.

Roche A, Girish B, Baere T, Baudin E, Boige V, Elias D, Lasser P, Schlumberger M \& Ducreux M 2003 Transcatheter arterial chemoembolization as first-line treatment for hepatic metastases from endocrine tumors. European Radiology V13 136-140.
La Rosa S, Sessa F, Capella C, Riva C, Leone BE, Klersy C, Rindi G \& Solcia E 1996 Prognostic criteria in nonfunctioning pancreatic endocrine tumours. Virchows Archiv 429 323-333.

Rougier P \& Mitry E 2000 Chemotherapy in the treatment of neuroendocrine malignant tumors. Digestion 62 73-78.

Skinazi F, Zins M, Menu Y, Bernades P \& Ruszniewski P 1996 Liver metastases of digestive endocrine tumours: natural history and response to medical treatment. European Journal of Gastroenterology \& Hepatology 8 673-678.

Sokmensuer C, Gedikoglu G \& Uzunalimoglu B 2001 Importance of proliferation markers in gastrointestinal carcinoid tumors: a Clinicopathologic Study. Hepatogastroenterology 48 720-723.

Strosberg JR, Choi J, Cantor AB \& Kvols LK 2006 Selective hepatic artery embolization for treatment of patients with metastatic carcinoid and pancreatic endocrine tumors. Cancer Control 13 72-78.

Sun W, Lipsitz S, Catalano P, Mailliard JA \& Haller DG 2005 Phase II/III study of doxorubicin with fluorouracil compared with streptozocin with fluorouracil or dacarbazine in the treatment of advanced carcinoid tumors: Eastern Cooperative Oncology Group Study E1281. Journal of Clinical Oncology 23 4897-4904 (DOI: 10.1200/JCO.2005.03.616).

Tomassetti P, Migliori M, Lalli S, Campana D, Tomassetti V \& Corinaldesi R 2001 Epidemiology, clinical features and diagnosis of gastroenteropancreatic endocrine tumours. Annals of Oncology 12 S95-S99.

Vilar E, Tabernero J, Casado E, Macarulla T, Ramos FJ, Martinelli E \& Saura C 2005 Lack of efficacy of streptozocin and doxorubicin in patients with advanced pancreatic endocrine tumors. American Journal of Clinical Oncology 28424.

Yasui H, Konishi E, Urasaki K, Yasukawa S \& Yanagisawa A 2006 Intratumoral heterogeneity of MIB-1 labelling index in gastric gastrointestinal stromal tumor (GIST). Gastric Cancer V9 223-228. 